\title{
Real-time monitoring and just-in-time intervention for adherence to pre-exposure prophylaxis among men who have sex with men in China: a multicentre RCT study protocol
}

Xia Jin ${ }^{1,2,3,4+}$, Hongyi Wang ${ }^{1,2,3,4+}$, Hang Li $1^{1,2,3,4}$, Zhenxing Chu ${ }^{1,2,3,4}$, Jing Zhang ${ }^{1,2,3,4}$, Qinghai Hu ${ }^{1,2,3,4}$, Wei Lv ${ }^{5}$, Xiaojie Huang ${ }^{6}$, Yaokai Chen ${ }^{7}$, Hui Wang ${ }^{8}$, Xiaoqing He $\mathrm{H}^{7}$, Lukun Zhang ${ }^{8}$, Zhili Hu ${ }^{1,2,3,4}$, Rantong Bao ${ }^{1,2,3,4}$, Shangcao Li $1^{1,2,3,4}$, Haibo Ding ${ }^{1,2,3,4}$, Wenqing Geng ${ }^{1,2,3,4}$, Yongjun Jiang ${ }^{1,2,3,4}$, Hong Shang ${ }^{1,2,3,4^{*}}$, Junjie $X u^{1,2,3,4^{*}}$ and for the CROPrEP Study Team

\begin{abstract}
Background: Pre-exposure prophylaxis (PrEP) is an effective biomedical strategy to prevent transmission of HIV infection, although medication adherence remains a challenge. We present the protocol for a multicentre randomised controlled trial to measure the effectiveness of a real-time monitoring and just-in-time intervention on medication adherence among PrEP users in China.

Methods: Study participants will include 1000 men who have sex with men (MSM) from four cites in China (Shenyang, Beijing, Chongqing and Shenzhen) attending a tenofovir disoproxil fumarate/emtricitabine (TDF/FTC) PrEP project as part of a real-world, prospective multicentre cohort study (CROPrEP). Participants will be randomised into the intervention and control arms in a 1:1 ratio. Participants in the intervention arm will be provided with remote real-time monitoring equipment that triggers twice just-in-time SMS (Short Messaging Service) medication reminders to PrEP users every half an hour when a scheduled dosage is missed, and followed with just-in-time SMS medication reminders to clinicians half an hour when there is no supplement after the second just-in-time SMS reminder to PrEP users. Clinicians will initiate individualised telephone intervention as soon as possible upon receipt of the just-in-time SMS missed dose alert. Those in the control arm will only receive generic weekly SMS reminders. The study will last 6 months. Participants will be seen at follow-up visits at three and 6 months. Trial outcomes to be measured include self-reported adherence assessed via questionnaire and pill counts, as well as drug concentration test results.

\footnotetext{
* Correspondence: hongshang100@hotmail.com; xjicmu@163.com

${ }^{+} \mathrm{Xia}$ Jin and Hongyi Wang contributed equally to this work.

'NHC Key Laboratory of AIDS Immunology (China Medical University),

National Clinical Research Center for Laboratory Medicine, The First Affiliated

Hospital of China Medical University, Shenyang 110001, China

Full list of author information is available at the end of the article
}

(c) The Author(s). 2020 Open Access This article is licensed under a Creative Commons Attribution 4.0 International License, which permits use, sharing, adaptation, distribution and reproduction in any medium or format, as long as you give appropriate credit to the original author(s) and the source, provide a link to the Creative Commons licence, and indicate if changes were made. The images or other third party material in this article are included in the article's Creative Commons licence, unless indicated otherwise in a credit line to the material. If material is not included in the article's Creative Commons licence and your intended use is not permitted by statutory regulation or exceeds the permitted use, you will need to obtain permission directly from the copyright holder. To view a copy of this licence, visit http://creativecommons.org/licenses/by/4.0/ The Creative Commons Public Domain Dedication waiver (http://creativecommons.org/publicdomain/zero/1.0/) applies to the data made available in this article, unless otherwise stated in a credit line to the data. 
(Continued from previous page)

Discussion: Medication adherence is critical to achieve optimal benefits from PrEP. This study will be the first individualised behaviour intervention using real-time technology to increase adherence among MSM PrEP users globally. If found effective, a real-time monitoring and just-in-time intervention system may be utilized for improving adherence and thus effectiveness of global PrEP application.

Trial registration: This study registered at ClinicalTrials.gov (ChiCTR1900025604) on September 2, 2019.

Keywords: HIV, men who have sex with men, Medication adherence, Pre-exposure prophylaxis, Internet of things, Electronic drug monitors

\section{Background}

Pre-exposure prophylaxis (PrEP) is recognized as highly effective in preventing new HIV infections in high-risk populations [1-9]. However, multiple clinical trials worldwide have shown that medication adherence to PrEP is suboptimal $[6,10,11]$, which reduces its effectiveness. The literature on medication adherence interventions provides several examples in the field of antiretroviral therapy (ART) and other clinical areas [12-14]. However, while scientific consensus on the efficacy of PrEP has been attained, there has been little work in development and evaluation of interventions to improve PrEP medication adherence.

Measuring adherence to PrEP is challenging and has traditionally been done using self-report questionnaires and by measuring pill counts and blood drug concentrations [15]. All of these methods are subject to limitations posed by the intermittent nature of data collection. For example, missed doses may be detected several weeks to months after they occur. In addition, interventions can only be conducted after poor medication adherence is detected by clinicians through follow-up interviews, pill counts, drug concentration, and adherence scales. Therefore, it is often impossible to initiate medication adherence interventions before viral rebound that would lead to treatment failure and drug resistance.

Electronic drug monitors (EDM) constitute a novel technology that has been applied in clinical practice for real-time adherence detection, reminder of adherence lapses, and interventions to resume treatment before treatment failure and onset of drug resistance [16-18]. The EDM system involves the use of a microchip embedded inside an intelligent pill container that records when the container is opened as an indication that the medication has been taken. A key innovation of advanced EDM technologies is to combine the Internet of Things with smartphone technology to monitor adherence in real time and provide personalised intervention. Patients can set their medication reminders (pillbox ringing and mobile phone reminders) to notify them when a scheduled dose is missed. The EDM system stores and uploads medication use data to support joint management by clinicians, the patient, and family members to improve medication adherence. Graphical adherence curves and scoring information can be generated on linked mobile phones, supporting real-time intervention from professionals when needed to avoid missed doses and overdoses.

This technology has been used to improve medication adherence of HIV treatment and other chronic diseases. However, the device has not yet been tested for PrEP. We now present a study protocol examining the use of EDM technology to monitor PrEP adherence in real time, along with personalised intervention to users to assess improvements in medication adherence with this system. This randomised controlled trial will prospectively assess the efficacy of this approach, providing scientific evidence to guide development of future PrEP intervention strategies.

\section{Methods \\ Objectives}

The objective of the study is to estimate the effectiveness of real-time monitoring and just-in-time intervention technology for improving PrEP medication adherence among Chinese MSM.

\section{Study setting}

The study includes four study centres (First Affiliated Hospital of China Medical University in Shenyang, Beijing You'An Hospital of Capital Medical University in Beijing, Shenzhen Third People's Hospital in Shenzhen, and Chongqing Public Health Medical Treatment Centre in Chongqing).

\section{Trial design}

This study is organized within the ongoing CROPrEP project, a multicentre, real-world prospective cohort study of daily or event-driven PrEP (emtricitabine/tenofovir disoproxil fumarate, TDF/FTC) among $1000 \mathrm{HIV}$ negative MSM most-at-risk of HIV infection in China. For the present study protocol, we randomly divided the 1000 CROPrEP study participants (500 from daily TDF/ FTC group and 500 from event-driven TDF/FTC group in the CROPrEP study) into intervention and control arms in a 1:1 ratio (250 in intervention arm and 250 in control arm for each of the original CROPrEP study groups). 


\section{Eligibility criteria}

The current RCT will be conducted with participants in the ongoing CROPrEP study. The inclusion criteria for the CROPrEP study include: (1) HIV-negative MSM; (2) aged 18-65 years; (3) report no severe damage to liver or kidney function; (4) self-report at least one of the following behaviours during the last 6 months: engaging in unprotected receptive anal sex with men, multiple male sexual partners, current or previous STIs (syphilis, gonorrhoea, chlamydia, chancroid, venereal lymphogranuloma, or other STI), had a history of post-exposure prophylaxis (PEP) but did not receive PEP in the last month; (5) not planning to leave local area of residence within the next 6 months (to ensure cohort retention); and (6) being able and agreeing to provide written informed consent. Individuals who maintained a monogamous sexual relationship with only one HIV-1 negative partner or one virologically suppressed HIV-1 positive partner for more than 1 year will be excluded.

\section{Interventions}

Participants in the intervention arm will be provided with a real-time monitoring device administering twice just-in-time SMS medication reminders to PrEP users every half an hour when a scheduled dosage is missed and followed with just-in-time SMS medication reminders to clinicians half an hour when there is no supplement after the second just-in-time SMS reminder to PrEP users. Clinicians will initiate individualised telephone intervention as soon as possible when they receive the just-in-time SMS medication reminder. Telephone interventions will be carried out at the same day for reminders received before 4:00 p.m. and 8:00 a.m. the next day for reminders received after 4:00 p.m., whereas control arm participants will receive general weekly SMS medication reminders. Participants in both groups will be invited to attend follow-up clinic visits at three and 6 months. The flow chart depicting the study design is shown in Fig. 1.

\section{Sample size}

We have powered the RCT to be able to detect meaningful changes in optimal adherence. Our estimates of outcome differences are based on our basic findings from our earlier pilot study with Chinese PrEP users, in which we found that proportions of adherence were 94\% versus $86 \%$, in intervention versus control patients after 6 months of the intervention. We will recruit 500 PrEP users per study arm. With the consideration of a twosided alpha of 0.05 and $40 \%$ loss to follow-up of our study, we will have a $90.6 \%$ power at to detect specified differences in adherence between the two arms after intervention.

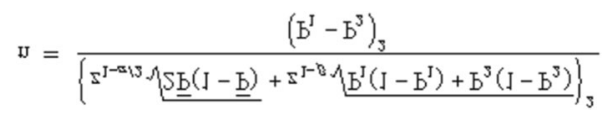

\section{Real-time monitoring equipment}

The medication adherence monitor (Msense, Icompanion Health Technologies, Guangzhou, China) records dosing behaviour in real time using microchips embedded in the container vial cap to detect when the container is opened (Fig. 2). This approach assumes that the participant takes the medication every time the monitored equipment is opened.

The real-time monitoring device can set the medication time, issue reminders for medicine to be taken, and trigger voice alerts for repeat doses. Data are automatically uploaded to a cloud-based server and can then be accessed by clinicians, PrEP users and family members via WeChat platform. Clinicians can monitor real-time data on PrEP use in the intervention arm from a backend platform and can provide just-in-time intervention to participants who did not take medicine according to prescription. PrEP users can also check their records on medication taken and not taken, to monitor their own medication usage and for reference during clinic visits. Graphical medication adherence curves and scoring data can be generated on mobile phones to support selfmanagement and personalised reminders. If participants in the intervention arm do not take medication on time according to prescription, clinicians and family members can send a message or initiate voice calls directly to the patient. Details on the intervention procedure are illustrated in Fig. 3.

\section{Randomisation}

Following the baseline study visit, all participants are randomised to one of the two study arms using 1:1 randomisation. The PASS 11.0 randomisation module will be used to ensure equal sizes of the two study groups. Each group will have 500 participants, and two groups of 500 random numbers will be generated for randomised grouping of the daily dose group and the eventdriven group to ensure equality of the two medication dosing strategies within the intervention arm (Table 1).

Accordingly, both the PrEP daily FTC/TDF group and the event-driven FTC/TDF group will have 250 PrEP users randomly assigned to the intervention arm and 250 assigned to the control arm. Recruitment targets of four study sites were determined based on patient volume and are shown in Table 2.

All study participants will be seen for a baseline study visit, followed up at 3 and 6 months, and fill an online self-administered questionnaire assessing sexual behaviours, psychological status, self-reported medication adherence, attitude and side effects towards PrEP use. 


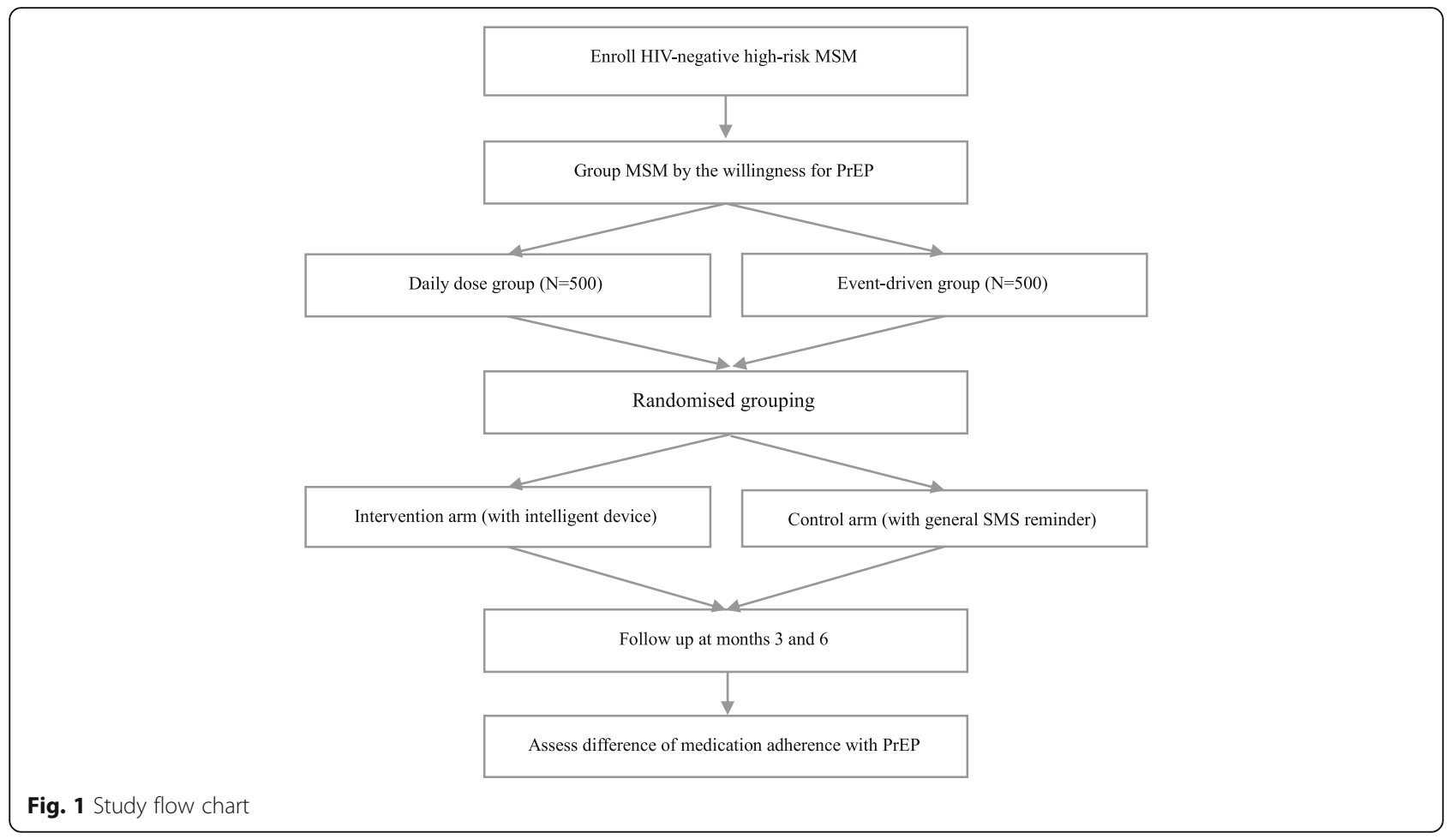

Blood samples and dry blood spots will be collected and tested as previously described for the CROPrEP project [19]. Pills remaining will be counted at the 3- and 6month follow-up visits, and refills will be provided to last until the next study or clinic visit. Participants will be required to return the real-time monitoring device provided by the program when the study is completed.

The program will incorporate locally trained community-based organizations (CBOs) serving MSM to recruit eligible participants for the study. $\mathrm{CBO}$ peer educators will be responsible for guiding the correct use of the monitoring device and will provide support services through social media platforms (WeChat, QQ). The study also offers incentives of US \$10 upon completion of the enrolment visit and US \$10 each at the 3- and 6-

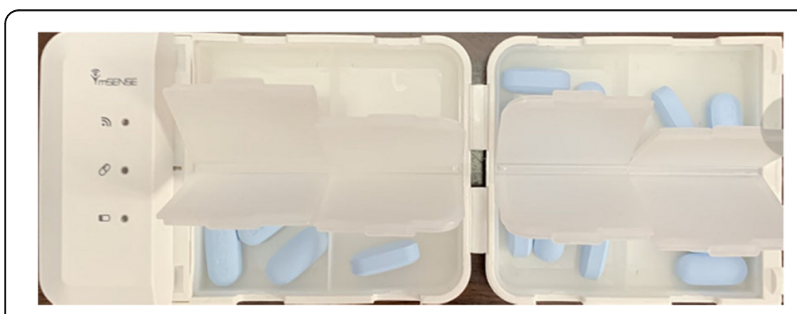

Fig. 2 Real-time monitoring device (Icompanion container) Note: The device was designed by Msense, Icompanion Health Technologies, Guangzhou, China. This figure is the real scene shooting by our CROPrEP Study Team. It was not derived from any published source and the copyright permissions for reuse is belonged to CROPrEP Study Team month visits (US $\$ 30$ total) to compensate the time contributed by participants.

\section{Laboratory procedure}

The laboratory testing procedures and parameters are described in the published protocol of the CROPrEP project [19]. MSM who are enrolled in the study and later screen as HIV positive will be instructed to discontinue PrEP and visit the local study hospital for HIV confirmation.

\section{Data collection and management}

The study questionnaire will be administered via a specialised online platform (Gold Data Technologies, Sichuan, China). Trained staff will collect data on remaining pill counts and verify test results.

$\mathrm{CBO}$ peer educators will instruct study participants on use of the real-time monitoring device at the baseline study visit and throughout follow-up using related images and videos in order to avoid overestimates (opening the container without removing pills) or underestimates (multiple pills removed during one opening of the monitoring device) of actual medication adherence. The monitoring device battery is sufficient to run in standby mode for 45 days after a 2 -h charge.

\section{Study endpoints}

Medication adherence refers to the degree to which a patient complies with the doctor's prescription to complete the treatment plan. It involves taking 


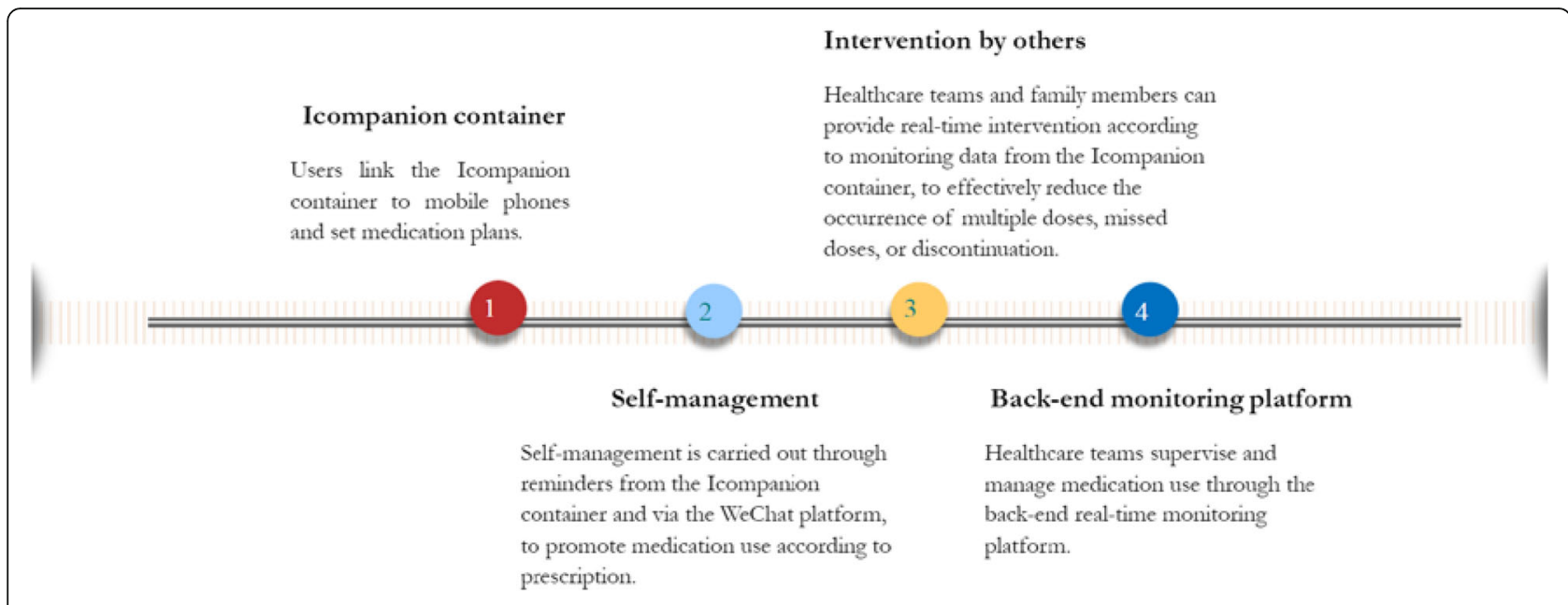

Fig. 3 Intervention procedure using a real-time monitoring device

medication regularly, not forgetting to take medication, and taking medication at the correct time, i.e., taking medicine according to the prescribed timing, dosage, and administration instructions. PrEP medication adherence will be measured via self-report questionnaires and pill counts as well as drug concentration test results. Details on the study endpoints are shown in Table 3.

\section{Statistical methods}

Normal distribution continuous variables will be presented by means and standard deviations while nonnormal distribution continuous variables will be presented by medians and interquartile ranges. For categorical variables, composition ratios and $95 \%$ confidence intervals will be used to describe the variables. Chi-square analysis will be used to assess differences between the study arms in HIV testing history, HIV self-testing (HIVST) characteristics, and high-risk behaviours. Multinomial logistic regression will be used to measure the outcome of real-time monitoring equipment on medication adherence.

\section{Ethics and dissemination}

Written informed consent will be provided to all study participants before take part in this study. The study was approved by the First Affiliated Hospital of
China Medical University Ethics Review Committee ([2019]2019-253-3).

\section{Discussion}

Traditional methods of measuring medication adherence such as self-report and pill counting are executed intermittently or at the end of the study period, often several months after the dosage has been missed. Accordingly, these methods often cannot guide intervention before the disease recurs or worsens. Hence, the ability to change medication adherence and prevent treatment failure or drug resistance with these methods is limited. In contrast, EDM is characterised by real-time monitoring and data processing, which allow healthcare teams and family members to monitor medication adherence and send reminders just-in-time to patients who have missed doses, before the disease may worsen. EDM is therefore a more efficient strategy to improve medication adherence.

Medication adherence is crucial for achieving optimal benefits from PrEP. However, many participants overreport PrEP medication adherence by traditional approaches such as self-reporting and pill counting, which adds to the challenge of accurately measuring medication adherence [15]. With traditional approaches,

Table 1 Illustration of randomisation procedure

\begin{tabular}{llll}
\hline Serial number & Group $^{\mathrm{a}}$ & Maximum deviation ratio to target (\%) & Cumulative sample size of two groups (A, B) \\
\hline 1 & B & $0.1 \%$ & $(0,1)$ \\
2 & A & $0.0 \%$ & $(1,1)$ \\
3 & A & $0.1 \%$ & $(2,1)$ \\
998 & B & $0.0 \%$ & $(499,498)$ \\
999 & A & $0.1 \%$ & $(500,499)$ \\
1000 & B & $0.0 \%$ & $(500,500)$
\end{tabular}

\footnotetext{
${ }^{a} A$, intervention arm; $B$, control arm
} 
Table 2 Number of participants to be recruited at each site

\begin{tabular}{lll}
\hline Sites & Intervention arm & Control arm \\
\hline Shenyang & 185 & 185 \\
Beijing & 210 & 210 \\
Shenzhen & 45 & 45 \\
Chongqing & 60 & 60 \\
Total & 500 & 500 \\
\hline
\end{tabular}

measurement of adherence after doses have been missed leads to an inability to provide timely intervention and thus to worse adherence. Real-time monitoring technology creates the ability to monitor medication adherence between routine visits and can trigger just-in-time intervention before adherence has lapsed for a substantial amount of time. Our study will be the first to directly compare traditional measurement of adherence versus real-time monitoring with just-in-time individualised intervention in PrEP users.

This study is focused on a novel, individualised behaviour intervention to reduce the risk of decreased medication adherence among PrEP users in China. Ours will be the first study of this type of intervention for improvement of PrEP medication adherence. Just-in-time telephone intervention requires investment of more time and energy. Our intervention employs novel technology combined with just-in-time SMS medication reminders and individualised telephone intervention to increase the intensity and efficiency of improving PrEP medication adherence in real time. Furthermore, telephone intervention only focuses on those with a missed dosage after just-in-time SMS reminders which can save time and energy. The control group will receive weekly universal undifferentiated SMS medication reminders since the real-time medication condition is unknown. Clinicians can provide just-in-time professional intervention based on information from the back-end real-time monitoring platform to improve the efficacy of PrEP in preventing the transmission of HIV infection. Dose-to-dose

Table 3 Study endpoints

\section{Medication adherence scores}

- The proportion of self-reported number of pills taken among the number of pills prescribed by the doctor.

- The proportion of the counted number of pills taken among the number of pills prescribed by the doctor.

- The proportion of pills taken as calculated by remote real-time monitoring equipment among the number of prescribed pills by the doctor.

Scores will be classified as high medication adherence ( $\geq 90 \%)$, intermediate medication adherence ( $\geq 60$ and $<90 \%$ ), or low medication adherence $(<60 \%)$

\section{Blood medicine concentration monitoring (TDF/FTC)}

Blood TDF/FTC concentration will be classified as high medication adherence ( $\geq 90 \%$ concentration), intermediate medication adherence $(\geq 60$ and $<90 \%)$, or low medication adherence $(<60 \%)$. medication adherence data and curves generated on adherence patterns are informative in promoting selfmanagement and intervention from others who are engaged with participants. The study has several important strengths, including capitalizing on the infrastructure of a large-scale, real-world multicentre study in China, use of real-time monitoring devices with just-in-time intervention to advance medication adherence, and measure the efficacy of such devices compared to traditional approaches.

Our study tests a real-time monitoring device with just-in-time intervention against other traditional approaches to address medication adherence in PrEP users. We hypothesize that by targeting real-time monitoring and intervention to reduce the risk of viral rebound, our strategy will improve TDF/FTC medication adherence, allowing clinicians and family members to maintain confidence regarding adherence.

Our study should be evaluated considering several limitations. First, the CROPrEP project is focused on the MSM population, in which medication adherence characteristics may be different from other patient populations. It has been shown that research study participants generally exhibit higher medication adherence levels than other clinical patients [20]. Accordingly, we recommend that future studies be conducted in other key populations to validate the effects of real-time monitoring and just-in-time intervention on PrEP medication adherence. In addition, the cost of real-time monitoring devices has limited their large-scale uptake in research and general clinical care. Thus, development of low-cost versions of these devices will be critically important for their future application.

\section{Abbreviations}

PrEP: Pre-exposure prophylaxis; MSM: Men who have sex with men; TDF/ FTC: Tenofovir disoproxil fumarate/emtricitabine; SMS: Short Messaging Service; ART: Antiretroviral therapy; EDM: Electronic drug monitors; HIVST: HIV self-testing

\section{Acknowledgments}

We would like to thank Yao Li, Fang Zhao for their efforts in the implementation of the project. We would also like to express our sincere appreciation to the study participants and community partners for their participation and support with recruitment for the study.

CROPrEP Study Team

China Medical University: Xiaoyun Shi, Rui Li, Yangyang Gao, Yanni Ma. Beijing Youan Hospital: Yi Duan, Guanghui Zhang. Chongqing Public Health Medical Center: Yao Li. Shenzhen Third People's Hospital: Fang Zhao.

\section{Authors' contributions}

HS and JX is responsible for all aspects of the study. XJ, HW, ZC and HL contributed to participant selection and IRB application. XH, YC, HW, XH, LZ, $J Z, Q H, Z H, R B, H D$ and LS performed the study. XJ drafted the manuscript. $J X, H W, Y J, W G$ and $H S$ reviewed the manuscript and provided edits and comments. WL and $\mathrm{XH}$ provided input into the study design. All authors have read and approve the final submission manuscript.

\section{Funding}

This work was supported by the Mega-Projects of National Science Research for the 13th Five-Year Plan (2017ZX10201101); the National Natural Science 
Foundation of China (81872674); National Science and Technology Major Project (2018ZX10101-001-001-003). None of the funders have had input into data collection, analysis, interpretation of the study results, or preparation of the manuscript.

\section{Availability of data and materials}

Not applicable.

\section{Ethics and approval and consent to participate}

The study was approved by the ethics review committee of the First Affiliated Hospital of China Medical University ([2019]2019-253-3) and registered at ClinicalTrials.gov (http://www.chictr.org.cn/index.aspx, ChiCTR1900025604) on September 2, 2019. All participants included in the study provided written informed consent.

\section{Consent for publication}

Not applicable.

\section{Competing interests}

The authors report no competing interests.

\section{Author details}

${ }^{1} \mathrm{NHC}$ Key Laboratory of AIDS Immunology (China Medical University), National Clinical Research Center for Laboratory Medicine, The First Affiliated Hospital of China Medical University, Shenyang 110001, China. ${ }^{2}$ Key Laboratory of AIDS Immunology, Chinese Academy of Medical Sciences, Shenyang 110001, China. ${ }^{3}$ Key Laboratory of AIDS Immunology of Liaoning Province, Shenyang 110001, China. ${ }^{4}$ Collaborative Innovation Center for Diagnosis and Treatment of Infectious Diseases, 79 Qingchun Street, Hangzhou 310003, China. ${ }^{5}$ Peking Union Medical College Hospital, Chinese Academy of Medical Sciences, Beijing 100730, China. ${ }^{6}$ Beijing Youan Hospital, Capital Medical University, Beijing 100069, China. ${ }^{7}$ Chongqing Public Health Medical Center, Chongqing 400036, China. ${ }^{8}$ Shenzhen Third People's Hospital, Shenzhen 518000, Guangdong, China.

Received: 20 March 2020 Accepted: 15 April 2020

Published online: 24 July 2020

\section{References}

1. McCormack S, Dunn DT, Desai M, et al. Pre-exposure prophylaxis to prevent the acquisition of HIV-1 infection (PROUD): effectiveness results from the pilot phase of a pragmatic open-label randomized trial. Lancet. 2016;387: 53-60.

2. Molina JM, Capitant C, Spire B, et al. On-demand preexposure prophylaxis in men at high risk for HIV-1 infection. N Engl J Med. 2015;373:2237-46.

3. Molina JM, Charreau I, Spire B, et al. Efficacy, safety, and effect on sexual behavior of on-demand pre-exposure prophylaxis for HIV in men who have sex with men: an observational cohort study. Lancet HIV. 2017;4:e402-10.

4. Baeten JM, Donnell D, Ndase P, et al. Antiretroviral prophylaxis for HIV prevention in heterosexual men and women. N Engl J Med. 2012;367(5): 399-410.

5. Choopanya K, Martin M, Suntharasamai P, et al. Antiretroviral prophylaxis for HIV infection in injecting drug users in Bangkok, Thailand (the Bangkok Tenofovir study): a randomised, double-blind, placebo-controlled phase 3 trial. Lancet. 2013;381(9883):2083-90.

6. Grant RM, Lama JR, Anderson PL, et al. Preexposure chemoprophylaxis for HIV prevention in men who have sex with men. N Engl J Med. 2010;363(27): 2587-99.

7. Marrazzo JM, Ramjee G, Richardson BA, et al. Tenofovir-based preexposure prophylaxis for HIV infection among African women. N Engl J Med. 2015; 372(6):509-18.

8. Thigpen MC, Kebaabetswe PM, Paxton LA, et al. Antiretroviral preexposure prophylaxis for heterosexual HIV transmission in Botswana. N Engl J Med. 2012;367(5):423-34.

9. Van Damme L, Corneli A, Ahmed K, et al. Preexposure prophylaxis for HIV infection among African women. N Engl J Med. 2012;367(5):411-22.

10. Molina JM, Capitant C, Spire B, et al. On demand PrEP with oral TDF-FTC in MSM: results of the ANRS Ipergay trial; Conference on Retroviruses and Opportunistic Infections; Seattle, Washington. 2015. Abstract 23LB.
11. Mugo PM, Sanders EJ, Mutua G, et al. Understanding adherence to daily and intermittent regimens of oral HIV pre-exposure prophylaxis among men who have sex with men in Kenya. AIDS Behav. 2015;19(5):794-801.

12. Mills $E$ J, Lester $R$, Thorlund $K$, et al. Interventions to promote adherence to antiretroviral therapy in Africa: a network meta-analysis. Lancet HIV. 2014;1: e104-11.

13. Audet CM, Salato J, Vermund SH, et al. Adapting an adherence support workers intervention: engaging traditional healers as adherence partners for persons enrolled in HIV care and treatment in rural Mozambique. Implement Sci. 2017;12(1):50.

14. Chaiyachati $\mathrm{KH}$, Ogbuoji O, Price $\mathrm{M}$, et al. Interventions to improve adherence to antiretroviral therapy: a rapid systematic review. AIDS. 2014; 28(Suppl 2):S187-204.

15. David S, Anna ME, Susanne S. A systematic review of adherence to oral preexposure prophylaxis for HIV - how can we improve uptake and adherence? BMC Infect Dis. 2018;18:581.

16. Lindsey EG, Jessica EH. Technological methods to measure adherence to antiretroviral therapy and preexposure prophylaxis. Curr Opin HIV AIDS. 2017:12(5):467-74.

17. Jessica EH, Josh $K$, Isaac $K$, et al. Real-time adherence monitoring for HIV antiretroviral therapy. AIDS Behav. 2010;14:1340-6.

18. William NR. Enhancing adherence in clinical research. Contemp Clin Trials. 2005;26:59-77.

19. Hongyi W, Yonghui Z, Zhu M, et al. Protocol for a multicenter, real-world study of HIV pre-exposure prophylaxis among men who have sex with men in China (CROPrEP). BMC Infect Dis. 2019:19:721.

20. Hughes DA, Bagust A, Haycox A, Walley T. The impact of non-compliance on the cost-effectiveness of pharmaceuticals: a review of the literature. Health Econ. 2001;10:601-15.

\section{Publisher's Note}

Springer Nature remains neutral with regard to jurisdictional claims in published maps and institutional affiliations.
Ready to submit your research? Choose BMC and benefit from:

- fast, convenient online submission

- thorough peer review by experienced researchers in your field

- rapid publication on acceptance

- support for research data, including large and complex data types

- gold Open Access which fosters wider collaboration and increased citations

- maximum visibility for your research: over $100 \mathrm{M}$ website views per year

At BMC, research is always in progress.

Learn more biomedcentral.com/submissions 